\title{
EDP scale analysis based on temperature
}

\author{
Xinyan Liu* \\ College of Computer Science and Technology,Tiangong University,Tianjin,300000
}

\begin{abstract}
Since the beginning of the 21st century,the global climate change,especially the global warming, is becoming more and more serious. The global warming leads to the rise of sea level,making many small island countries at a low altitude in danger of vanishment.Firstly,we build an EDP formation model to predict which countries and how many people will have the risk of becoming EDP.We use principal component analysis to reveal that EDP is mainly caused by the rise of sea level.We choose a time series prediction algorithm,fbprophet to predict the trend of temperature change.Based on the rise of sea level,the size of EDP affected by natural disasters is roughly estimated.
\end{abstract}

Keywords: EDP;Fbprophet

\section{Introduction}

In the 21st century,natural disasters have swept over most countries and regions in the world;and sea level rise is one of the significant manifestations. Sea-level rise caused by global warming has been a concern of countries around the world.The possible impacts of sea-level rise include flooding lowlands, exacerbating coastal erosion,increasing the frequency of storm surges, and saltwater intrusion.However, the small island nations scattered in vast oceans,located in the Indian Ocean and Pacific Ocean are the most vulnerable victims of climate change.How to settle these people displaced by environmental changes so as to protect the human rights of these EDPs as much as possible and reduce the loss of cultures are also questions worth pondering.

\section{EDP Formation Model}

\subsection{Global Climate Change Analysis}

There are many climatic reasons for the emergence of EDP,including high sea level flooding land and areas at low altitudes along the coast, the climate is no longer suitable for human survival,or a series of natural phenomena caused by abnormal ocean conditions may cause some human Displaced by environmental changes. We chose to use survey data to determine the main factors affecting the formation of EDP by applying principal component analysis to the causes of these EDPs.Principal Component Analysis can avoid reducing the information contained in the original target as much as possible.Through the method of dimensionality reduction, a comprehensive index can be found to roughly determine the main reasons affecting the subject,thereby determining the main reasons for EDP.

Principal Component Analysis

The purpose of PCA is to use the idea of dimensionality reduction to concentrate the information scattered on a set of variables to a few comprehensive variables(linear combinations), and the obtained comprehensive variables are not related to each other.

PCA is to synthesize the original $\mathrm{P}$ observation variables $x_{1}, x_{2} \ldots x_{p}$ to form $\mathrm{P}$ new variables(composite variables), that is:

$$
F_{i}=w_{1 i} x_{1}+w_{2 i} x_{2}++\mathbf{w} \mathbf{x}_{p i} \quad,, i=1,2 \ldots p
$$

Here, $x_{i}$ is an $\mathrm{n}$-dimensional vector,and $F_{i}$ is also an n-dimensional vector.The coefficient $w_{i j}$ of the above model must meet the following three conditions:

(1) $F_{i}$ is not related to $F_{j}(i \neq j ; i, j=1,2 \ldots p)$

(2)The variance of $F_{1}$ is greater than the variance of $F_{2}$ is greater than the variance of $F_{3}$, and so on

(3) $w_{k 1}^{2}+w_{k 2}^{2}+\ldots+w_{k p}^{2}=1, k=1,2 \ldots p$

When the above three conditions are met, the new random variables obtained by the transformation are not correlated with each other,and the variance decreases in order.

\subsection{Predict Sea Level Rise and Determine Who Might Become EDP}

Copyright (C) 2020 Xinyan Liu

doi: 10.18282/1-e.v9i4.1696

This is an open-access article distributed under the terms of the Creative Commons Attribution Non-Commercial License (http://creativecommons.org/licenses/by-nc/4.0/), which permits unrestricted non-commercial use, distribution, and reproduction in any medium, provided the original work is properly cited. 
We have determined the main cause of Environmentally Displaced Persons(EDP)is the change in geographic conditions due to global climate change. That is, the rising temperature brought by global warming has caused seawater expansion and melting of glaciers. This is the most important factor that causes sea level to rise year by year,and this has caused some island nations at lower altitudes to lose their living space.Displaced with it.They have become homeless.According to the latest atmospheric temperature data from the US National Oceanic and Atmospheric Administration and the latest sea level data from the Commonwealth Scientific and Industrial Research Organization of Australia,we first use Facebook time series prediction algorithm fbprophet to predict the temperature trend.

2.2.1 Forecasting Air Temperature Using Time Series Prediction Algorithm Fbprophet

The prophet algorithm can not only handle some outliers in the time series,but also some missing cases.It can predict the future trend of the time series almost automatically.The fast iterative optimization of the time series model is presented.Prophet's algorithm implementation:

In the field of time series analysis, there is a common analysis method called the Decomposition of Time Series, which divides the time series into several parts,namely the seasonal term, the trend term, and the remaining term.In other words,for all

$$
y_{t}=S_{t}+T_{t}+R_{t}
$$

In addition to the form of addition, there is also the form of multiplication, which is:

$$
y_{t}=S_{t} \times T_{t} \times R_{t}
$$

Generally speaking,in real life and production,in addition to seasonal terms,trend terms,and residual terms, there are usually the effects of holidays. Therefore, in the prophet

algorithm, the experts considered the above four items at the same time, that is:

$$
y(t)=g(t)+s(t)+h(t)+\varepsilon_{t}
$$

Where ${ }^{g(t)}$ represents the trend term, which represents the change trend of the time series above the aperiodic; $s(t)$ represents the period term,or seasonal term, which is generally based on weeks or years; $h(t)$ represents a holiday item,indicating whether there is a holiday on the day,but we didn't use it this time; $\varepsilon(t)$ represents the error term or the remaining term.

Prophet's workflow is as shown in the figure above.By integrating the two modules of modeling and evaluation,fast iterative optimization of the time series model is realized.

2.2.2 Forecasting Future Sea Level Changes Through Predicted Temperature Changes

Through the previous analysis,we predicted the changes in temperature with the year and the relationship between temperature and sea level.We can combine the two to predict the future changes in sea level from the changes in temperature.

\subsection{Scale of Affected People}

With the rise of sea level, it will not only submerge some islands with low altitude,but also bring natural disasters such as coastal erosion,salt intrusion into soil,fresh water,storm surge,etc.,which seriously threaten the survival of indigenous people,making them have to leave their hometown to find new accommodation.By 2050,150 million people will be below the water line at high tide,300 million people will live in flooded areas every year,and tens of millions of people will be displaced in the next decades of this century.Island countries,Delta areas with relatively large population and coastal countries are likely to disappear from the earth due to sea-level rise.

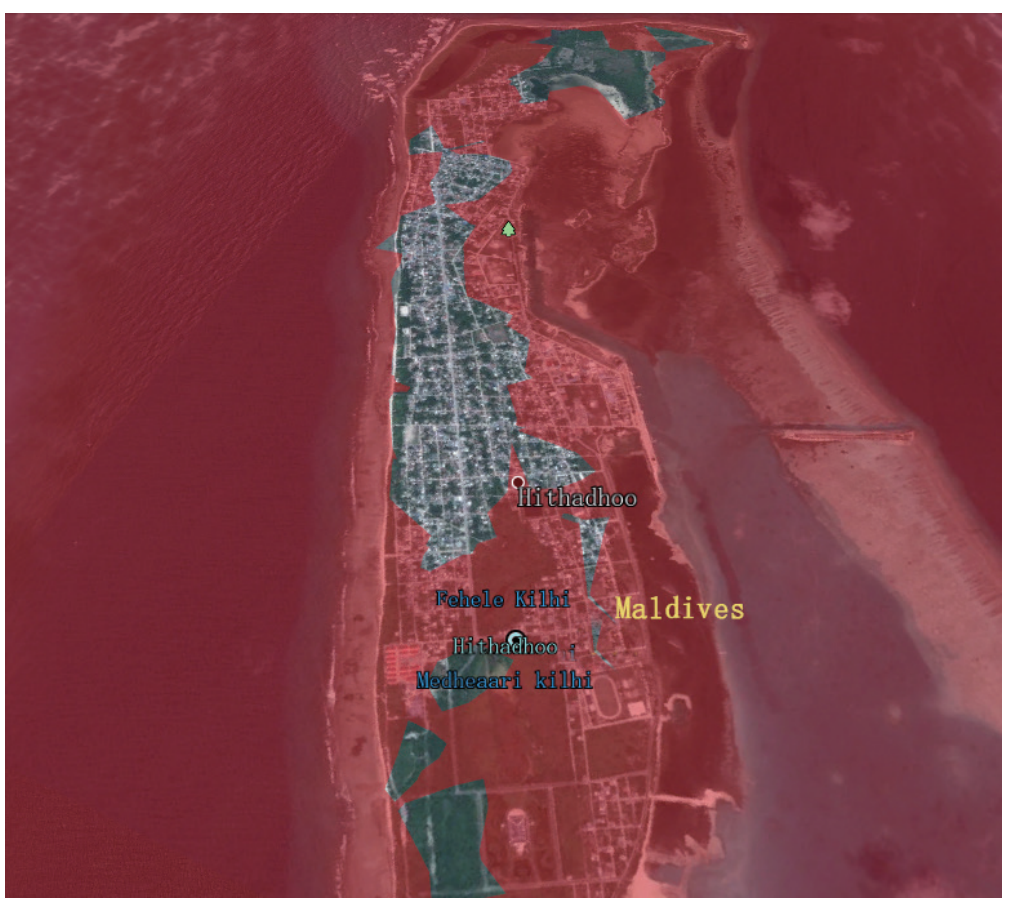

Figure 1: What Maldives looks like in 2100 


\title{
Application of Computer Graphic Technology in Animated Scene
}

\author{
Changyong Zhu , yunyun Wang , J iaxin Tang \\ Nantong Institute of Technology Nantong, Jiangsu 226002
}

Abstract:Animated scene design directedly refers to other styling design besides character design, which plays extremely important role in animated films. Scenes usually vary with plots of story, highlighting atmosphere and style of animation. Therefore, design of animated scene conveys characteristics of both technology and art. This article illustrates the specific application of computer graphic technology in animated scene, featuring the animation, the man on the stage, made by self.

Keywords:Animated scene, three dimentional animation, Peking Opera.

With the rapid development of digital media technology, the traditional two-dimensional animation scene can no longer meet people's visual needs. To cater to audiences, we could create three-dimensional space by using graphic and image technology in which we can make 3D animation with the sense of space and reality. To be more specific, we can build 3d model, set up material and texture, simulate realistic lightening, rig models and set up rendering to reach that purpose.

Three-dimensional animation has been widely spread in various fields. Specifically, in the field of digital games, 3D animation technology enables users to immerse themselves in the wonderland of virtual game world. In the field of digital entertainment, animation made with 3D technology seems to be more appealing, with its various styles of animated scene and distinctive characters. In the field of military, realistic battle scene could be simulated using 3D technology. Compared with 2D graphics, 3D graphics seem to be more vivid and stereoscopic, which are more likely to enhance audiences' visual comprehension. This measure benefits the creators of animation in their intention, bringing about a better experience to audience with more immersion and sense of realism.

Peking opera plays an important position in the long history of Chinese history. However, Peking opera culture is gradually weakening with the flowing of time, during which the birth, dan, net, end, and ugliness in Peking opera are gradually forgotten. Therefore, to protect this form of cultural heritage, three-dimensional animation is highly recommended, which depicts realistic scenery and provides a novel visual experience. In this animated short film, the ancient theater building group is used as a reference object. To present a unique architecture style, re-designing of Chinese ancient architecture is conducted during the production of animation On The Stage. The production of the animation scene combines both technology and art.

The production procedure of the animated film On the Stage is consisted of eight steps, which is summarized as scene design according to the script, research and study, redesign and planning of scene, 3D environment design and modeling, story broad planning, lightening and rendering, composition and postproduction. The animated film On the Stage will be introduced in this article.

\section{Story\& Background}

Peking opera, as an expression of traditional Chinese art form, conveys its spectacular expression and charm, staying popular among people who fancy art in the whole world range. There is one plot in the story that the performer who is making up behind the stage recalling the memory when he was performing on the stage with crowds of audience immersed in the performance. "Lan

Copyright (C) 2020 Zhu Changyong et al.

doi: $10.18282 / 1-$ e.v9i4.1697

This is an open-access article distributed under the terms of the Creative Commons Attribution Non-Commercial License (http://creativecommons.org/licenses/by-nc/4.0/), which permits unrestricted non-commercial use, distribution, and reproduction in any medium, provided the original work is properly cited.

\section{Conclusion}

Through the analysis of the above-mentioned number of affected people,we found that more and more countries and populations will be affected by environmental changes. The scale of EDP will become larger and larger,and the cultural loss caused by it will be inevitable.In this regard,our team has established a cultural loss risk assessment model to assess the continuous impact of EDP on cultural loss.

\section{References}

[1]Jason D.Söderblom(2008).Climate Change:National\&Regional Security Threat Multiplier for Australia[J].SECURITY SOLUTIONS.Pp.60-68.

[2]Emma Brindal(2007),Asia-Pacific:Justice for Climate Refugees[J].pp.240

[3]UN, The judgment of Huber in the Island of Palmas case 2 UN Doc RIAA829/838(1928) 